\section{Brain and Brawn: Role of Exercise-Induced Myokines}

\author{
Jun Hyun Bae', Wook Song ${ }^{1,2, *}$ \\ ${ }^{1} \mathrm{Health}$ and Exercise Science Laboratory, Institute of Sport Science and ${ }^{2}$ Institute on Aging, Seoul National \\ University, Seoul, Korea
}

\author{
Received June 5, 2019 \\ Reviewed June 26, 2019 \\ Accepted July 11, 2019 \\ ${ }^{*}$ Corresponding author \\ Wook Song
}

https://orcid.org/0000-0002-8825-6259

Health and Exercise Science Laboratory, Institute of Sport Science, Seoul National University, 1 Gwanak-ro, Gwanak-gu, Seoul 08826 Korea

Tel: +82-2-880-7791

Fax: +82-2-872-2867

E-mail: songw3@snu.ac.kr
As the rate of aging populations in the world has increased, there has been a growing interest in preventing and delaying aging. A multidisciplinary study has been conducted in several areas, and it has been found that physical activity or exercise is an essential factor that positively contributes to the prevention and delay of agerelated diseases such as geriatric disease and frailty. ${ }^{1}$

Ability to maintain physical function is associated with exerciseinduced skeletal muscle metabolism and ability, and particularly skeletal muscle communicates with other organs through the production and release of myokines during exercise. These exercise-induced myokines have mostly positive effect of overall health-related outcome, and there are few negative effects such as proinflammatory effect, proatherogenic actions, and increasing insulin resistance. ${ }^{2}$ However, many studies report that exercise has a positive effect on cognitive function in neurological disease, including dementia. ${ }^{3}$ Thus, exercise has a significant impact on overall skeletal muscle function and geriatric disease, including sarcopenia, frailty, and cognitive function.

Exercise-induced myokines show improvements in physical function and skeletal muscle metabolism in aging populations. For instance, 50 women over the age of 65 , who participated in 12 weeks of resistance exercise, improved their isokinetic leg strength, grip strength, and irisin (a myokines) levels. ${ }^{4}$ Moreover, secreted protein acidic and rich in cysteine (SPARC) was ameliorated by moderated steady-state cycling exercise. ${ }^{5}$ A myonectin was also decreased after 10 weeks of moderate aerobic exercise in healthy older women. ${ }^{6}$ These results raised the possibility that the benefit of exercise on physical function are mediated by myokine signaling, but the results were contractive. Moreover, only a limited number of clinical trial results attribute aerobic training and outcomes to myokines, and those observations of enhanced physical function do not accurately explain older adults' performance. Thus, it is still necessary to investigate the relationship between cardiovascular function, muscular strength, and resistance training to determine effective interventions for increasing myokine levels via muscle regeneration for preventing age-related decline in muscle function. Although there exist beneficial results on myokines in animals, it currently lacks adequate understanding of the validity of those results for humans and the physiological basis of myokine changes in humans.

Age-related frailty and cognitive function are closely related to each other. Of note, the concept of "cognitive frailty" is used to define the presence of both physical frailty and potentially reversible cognitive impairment in the absence of dementia. ${ }^{7}$ One study 
showed that regular exercise can maintain and improve brain functions in older adults who were experiencing cognitive frailty. ${ }^{7}$ In other words, physical frailty and sarcopenia are affected by the cognitive function. Research has also showed that exercise-induced myokines can improve cognitive function. For example, in one study, the myokine brain-derived neurotrophic factor (BDNF) increased after exercise and benefitted cognitive function. ${ }^{8}$ In another study, treadmill exercise increased human plasma level of cathepsin B, which mediated effects on cognitive function. ${ }^{9}$ Circulation of irisin was also improved by endurance exercise, ${ }^{10}$ but its effects on cognitive function were inconclusive. Association of exercise and cognitive function in older adults was investigated and 12 weeks of high-speed exercise training enhanced cognitive function, especially in digit span forward test. ${ }^{11}$

Similarly, 16 weeks of resistance training reduced older adults' level of physical frailty and cognitive impairment. ${ }^{12}$ These results corroborate the hypothesis that exercise would positively affect cognitive function and raise the possibility of the mechanism in which exercise-induced myokines (e.g., BDNF, irisin, insulin-like growth factor-1, and cathepsin B) $)^{13,14}$ play an important role in cross-talk between brain and skeletal muscle. Thus, it is highly speculated that skeletal muscle secrets myokines which can alter cognitive function through the effect of peripheral factors via autocrine and paracrine manners. However, it is necessary to elucidate underlying molecular mechanism to explain the detail cross-talk story between two organs brain (cognition) and skeletal muscle (physical function). In addition, it needs to clarify how exercise affects brain structure morphologically and correspondent function in humans with imaging study.

In conclusion, exercise-induced myokines have profound effects on both skeletal muscle and the brain, and they are associated with those molecular and cellular mechanistic changes in geriatric diseases such as sarcopenia, frailty, and cognitive frailty. This area of research will enable us to understand the potential endocrine crosstalk between skeletal muscle and brain, ${ }^{15}$ which could explain "brain and brawn" better.

\section{CONFLICTS OF INTEREST}

The authors declare no conflict of interest.

\section{AUTHOR CONTRIBUTIONS}

Study concept and design: WS; drafting of the manuscript: all authors; critical revision of the manuscript: all authors; administrative, technical, or material support: WS; and study supervision: WS.

\section{REFERENCES}

1. McPhee JS, French DP, Jackson D, Nazroo J, Pendleton N, Degens H. Physical activity in older age: perspectives for healthy ageing and frailty. Biogerontology 2016;17:567-80.

2 . Fuster JJ, Walsh K. The good, the bad, and the ugly of interleukin-6 signaling. EMBO J 2014;33:1425-7.

3. Gomez-Pinilla F, Hillman C. The influence of exercise on cognitive abilities. Compr Physiol 2013;3:403-28.

4. Kim HJ, So B, Choi M, Kang D, Song W. Resistance exercise training increases the expression of irisin concomitant with improvement of muscle function in aging mice and humans. Exp Gerontol 2015;70:11-7.

5. Aoi W, Naito Y, Takagi T, Tanimura Y, Takanami Y, Kawai Y, et al. A novel myokine, secreted protein acidic and rich in cysteine (SPARC), suppresses colon tumorigenesis via regular exercise. Gut 2013;62:882-9.

6. Lim S, Choi SH, Koo BK, Kang SM, Yoon JW, Jang HC, et al. Effects of aerobic exercise training on $\mathrm{C} 1 \mathrm{q}$ tumor necrosis factor $\alpha$-related protein isoform 5 (myonectin): association with insulin resistance and mitochondrial DNA density in women. J Clin Endocrinol Metab 2012;97:E88-93.

7. Yoon DH, Lee JY, Shin SA, Kim YK, Song W. Physical frailty and amyloid- $\beta$ deposits in the brains of older adults with cognitive frailty. J Clin Med 2018;7:E169.

8. Szuhany KL, Bugatti M, Otto MW. A meta-analytic review of the effects of exercise on brain-derived neurotrophic factor. J Psychiatr Res 2015;60:56-64.

9. Moon HY, Becke A, Berron D, Becker B, Sah N, Benoni G, et al. Running-induced systemic cathepsin B secretion is associated with memory function. Cell Metab 2016;24:332-40.

10. Miyamoto-Mikami E, Sato K, Kurihara T, Hasegawa N, Fujie S, Fujita $S$, et al. Endurance training-induced increase in circulat- 
ing irisin levels is associated with reduction of abdominal visceral fat in middle-aged and older adults. PLoS One 2015;10: e0120354.

11. Yoon DH, Kang D, Kim HJ, Kim JS, Song HS, Song W. Effect of elastic band-based high-speed power training on cognitive function, physical performance and muscle strength in older women with mild cognitive impairment. Geriatr Gerontol Int 2017;17:765-72.

12. Yoon DH, Lee JY, Song W. Effects of resistance exercise training on cognitivefunction and physical performance in cogni- tive frailty: a randomized controlled trial. J Nutr Health Aging 2018;22:944-51.

13. Kim S, Choi JY, Moon S, Park DH, Kwak HB, Kang JH. Roles of myokines in exercise-induced improvement of neuropsychiatric function. Pflugers Arch 2019;471:491-505.

14. Pedersen BK. Physical activity and muscle-brain crosstalk. Nat Rev Endocrinol 2019;15:383-92.

15. Delezie J, Handschin C. Endocrine crosstalk between skeletal muscle and the brain. Front Neurol 2018;9:698. 